\title{
The Two Faces of the Liquid Ordered Phase
}

Itay Schachter, ${ }^{\dagger,+}$ Riku O. Paananen, ${ }^{\S}$ Balázs Fábián, ${ }^{\dagger}$ Piotr Jurkiewicz, ${ }^{*}, \|$ and Matti Javanainen ${ }^{*},, \perp$

$\dagger$ Institute of Organic Chemistry and Biochemistry of the Czech Academy of Sciences, Flemingovo nám. 542/2, CZ-16000 Prague 6, Czech Republic

$\ddagger$ Institute of Chemistry, the Fritz Haber Research Center, and the Harvey M. Kruger center for Nanoscience 83 Nanotechnology, The Hebrew University, Jerusalem 9190401, Israel

I These authors contributed equally.

$\S$ Helsinki Eye Lab, Department of Ophthalmology, University of Helsinki and Helsinki University Hospital, FI-00014 University of Helsinki, Finland

\|J. Heyrovský Institute of Physical Chemistry of the Czech Academy of Sciences, Dolejškova 2155/3, CZ-18223 Prague 8, Czech Republic

$\perp$ Institute of Biotechnology, University of Helsinki, FI-00014 University of Helsinki, Finland

E-mail: piotr.jurkiewicz@jh-inst.cas.cz; matti.javanainen@helsinki.fi 


\begin{abstract}
The coexistence of liquid ordered $\left(\mathrm{L}_{\mathrm{o}}\right)$ and liquid disordered $\left(\mathrm{L}_{\mathrm{d}}\right)$ phases in synthetic and plasma membrane-derived vesicles serves as a model for biomembrane heterogeneity. However, the connection between the structures of microscopic phases present in vesicles at low temperatures and the tiny ordered "raft" domains of biomembranes at body temperature is unclear. To study the $\mathrm{L}_{\mathrm{o}}$ phase structure across temperatures, we performed atomistic molecular dynamics simulations, differential scanning calorimetry, and fluorescence spectroscopy on the $\mathrm{L}_{\mathrm{o}}$ phase in binary and ternary lipid mixtures. Our results reveal an $\mathrm{L}_{\mathrm{o}}$ phase with highly ordered and hexagonally packed clusters of saturated lipid chains at low temperatures. These clusters melt upon heating, and numerous membrane properties reflect this transition as two regimes with different temperature dependence. Still, the transition between the regimes is continuous, and they both match the description of the $\mathrm{L}_{\mathrm{o}}$ phase with high order and relatively high mobility. Our findings question the use of vesicles displaying $\mathrm{L}_{\mathrm{o}}-\mathrm{L}_{\mathrm{d}}$ coexistence as models for heterogeneity in cellular membranes, as they likely correspond to different molecular organizations.
\end{abstract}

\title{
Graphical TOC Entry
}

\begin{tabular}{|l|}
\hline TOC ENTRY REQUIRED \\
\hline
\end{tabular}


When inserted into certain phospholipid membranes in sufficient amounts, cholesterol (CHOL) induces the liquid ordered $\left(\mathrm{L}_{\mathrm{o}}\right)$ phase, ${ }^{1}$ whose properties are intermediate between the liquid disordered $\left(\mathrm{L}_{\mathrm{d}}\right)$ and gel phases: acyl chains display gel-like ordering, whereas rotational and translational diffusion is relatively fast akin to the $\mathrm{L}_{\mathrm{d}}$ phase. CHOL increases order, thickness, and stiffness of the $\mathrm{L}_{\mathrm{d}}$ phase, yet has the opposite effects on the gel phase. ${ }^{2}$ Therefore, binary mixtures of CHOL and certain phospholipids have a threshold CHOL concentration, above which the $\mathrm{L}_{\mathrm{d}}-$ gel transition vanishes. For dipalmitoylphosphatidylcholine (DPPC) this happens at $\approx 25-30 \mathrm{~mol} \%$ of CHOL, above which the membrane properties show continuous temperature dependence, indicating a uniform $\mathrm{L}_{\mathrm{o}}$ phase. This behavior is reported by numerous experimental approaches ${ }^{3-8}$ and simulations. ${ }^{9-12}$ Still, some studies have reported nonidealities within the $\mathrm{L}_{\mathrm{o}}$ region: differential scanning calorimetry (DSC) and NMR detected a broad transition, ${ }^{13,14}$ which was suggested to correspond to the transition between two distinct $\mathrm{L}_{\mathrm{o}}$ phases ${ }^{14}$ differing in lipid chain tilt. ${ }^{14,15} \mathrm{NMR}$ spectroscopy and X-ray scattering suggested that even at high CHOL concentrations, heating melts the glycerol region, shifts CHOL towards lipid headgroups, and disorders acyl chain termini. ${ }^{16,17}$

Certain ternary mixtures of low- $T_{\mathrm{m}}$ and high- $T_{\mathrm{m}}$ lipids and CHOL display $\mathrm{L}_{\mathrm{o}} / \mathrm{L}_{\mathrm{d}}$ coexistence, ${ }^{18}$ modelling the putative lipid domains ("rafts") in biomembranes. ${ }^{19}$ The coexistence is visible at temperatures below the $T_{\mathrm{m}}$ of the high- $T_{\mathrm{m}}$ lipid, and similarly in giant plasma membrane-derived vesicles well below the body temperature. ${ }^{20}$ This agrees with CHOL's ability to melt the gel phase, and recent simulations have indeed demonstrated ordered phases with hexagonally-packed and CHOL-depleted regions, ${ }^{21-23}$ despite CHOL's preference for saturated chains. ${ }^{24}$ However, it is unclear whether the $\mathrm{L}_{\mathrm{o}}$ phase observed in phase-separated vesicles structurally differs from that observed at higher $T$, and whether the former is a faithful model plasma cell membrane heterogeneity.

Experiments have struggled to provide a consistent molecular view of the $\mathrm{L}_{\mathrm{o}}$ phase in binary mixtures, and the situation is equally complicated for ternary mixtures: $\mathrm{NMR}^{25}$ and X-ray scattering ${ }^{26}$ resolve signals originating from the two coexisting phases, yet their 
compositions are temperature-dependent.

To overcome these limitations, we performed differential scanning calorimetry measurements and fluorescence experiments of binary and ternary lipid mixtures corresponding to the $\mathrm{L}_{\mathrm{o}}$ phase, and provided a molecular picture of the observed phenomena with atomistic molecular dynamics simulations. We studied bilayers consisting of 1) a ternary mixture consisting of $55 \mathrm{~mol} \% \mathrm{DPPC}, 15 \mathrm{~mol} \% \mathrm{DOPC}$, and $30 \mathrm{~mol} \% \mathrm{CHOL}$, corresponding to the composition of the $\mathrm{L}_{\mathrm{o}}$ phase of a phase-separated $0.40 / 0.40 / 0.20$ mixture at $298 \mathrm{~K}{ }^{21,27} \mathrm{Upon}$ heating, this mixture remains in the $\mathrm{L}_{\mathrm{o}}$ phase. ${ }^{27,28}$ 2) A binary mixture of $70 \mathrm{~mol} \% \mathrm{DPPC}$ and $30 \mathrm{~mol} \% \mathrm{CHOL}$, which falls to the $\mathrm{L}_{\mathrm{o}}$ regime at all relevant temperatures. ${ }^{8}$

The simulations revealed distinct changes in bilayer structure depending on the temperature, as shown in Fig. 1 (see Figs. S1 and S2 for more temperatures). At 293 K, DPPC chains are arranged in all-anti conformation, whereas at $333 \mathrm{~K}$ they are predominantly in gauche conformation in both binary and ternary mixtures. At $313 \mathrm{~K}$, their behaviors differ with the former showing more ordered chain conformations. This indicates that DPPC undergoes some kind of a transition yet at different temperatures for the two mixtures. The question is whether this transition is visible in membrane properties.

It indeed is, as demonstrated in Fig. 2. Area per phospholipid (APPL), shown in Fig. 2A), exhibits two regimes with different thermal expansion coefficients without a large jump yet a crossover at $T_{\mathrm{co}}^{\mathrm{ter}} \approx 308 \mathrm{~K}$ (ternary mixture) or $T_{\mathrm{co}}^{\mathrm{bin}} \approx 318 \mathrm{~K}$ (binary mixture). These coefficients also differ between the binary and ternary mixtures in both temperature regimes. The ternary mixture contains fluid DOPC, which explains the slightly larger coefficient below $T_{\text {co. }}{ }^{29}$

Differential scanning calorimetry (DSC) is the tool of choice to detect phase transitions or more subtle molecular rearrangements. We performed such measurements on unilamellar vesicles for the binary and ternary mixtures (see Methods and SI). As shown in Figs. 4A, DSC detects broad peaks for the binary and ternary systems, and their maxima agree perfectly with our simulations (see labels in Fig. 4A). This suggests that a pure $\mathrm{L}_{\mathrm{o}}$ phase is present at 

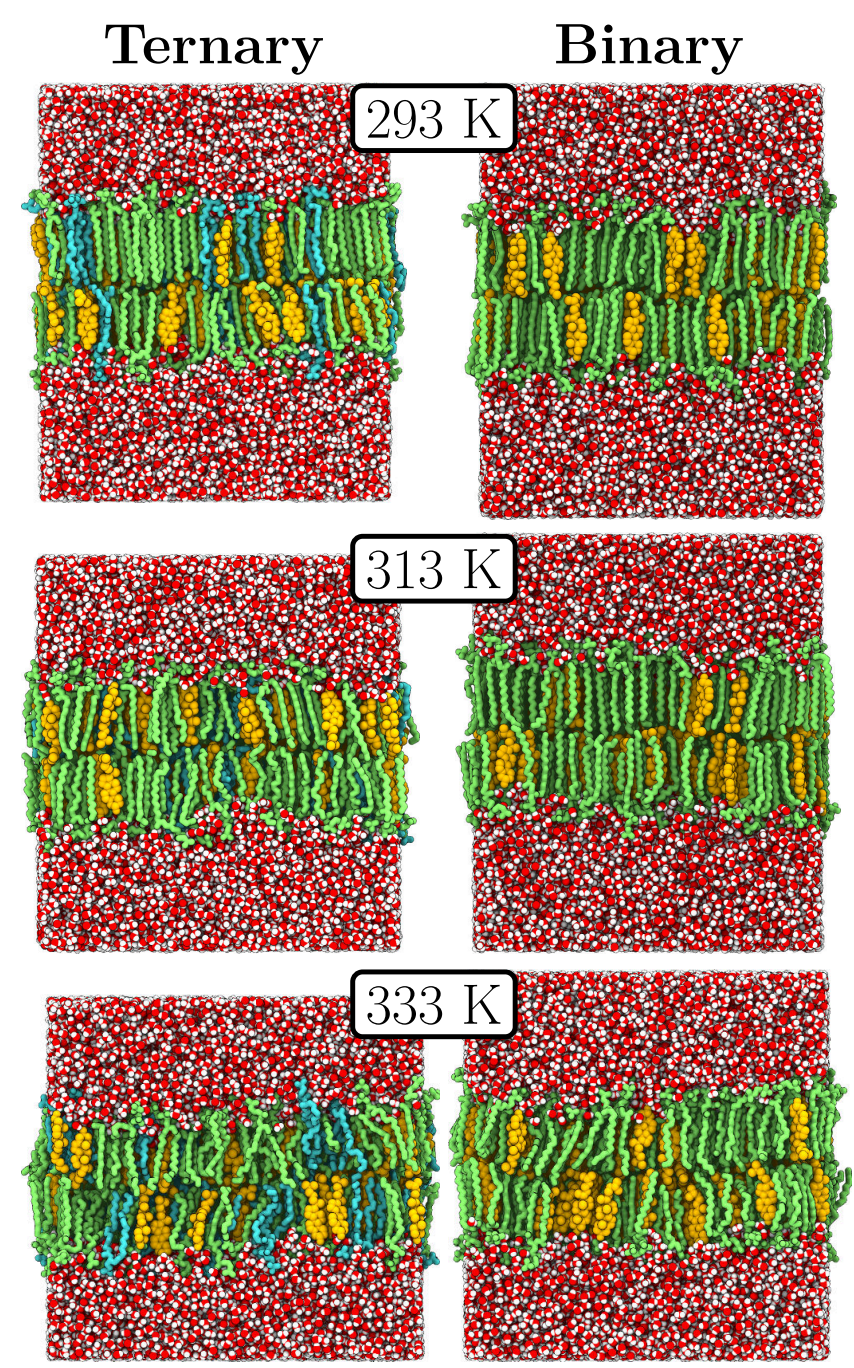

Figure 1: Final structures (after 1 us of simulation) of ternary (left) and binary (right) mixtures at $293 \mathrm{~K}$ (top), $313 \mathrm{~K}$ (middle), and $333 \mathrm{~K}$ (bottom). The chains are in all-anti conformation at $293 \mathrm{~K}$, yet they melt fully at $323 \mathrm{~K}$. Here, DPPC is shown in green, DOPC in blue, and CHOL in yellow. Lipid hydrogens and ions are omitted. Water is shown in red and white.

at all $T$, yet it undergoes a minor structural transition. Measurements on pure DPPC reveal a gel-liquid transition at $T_{\mathrm{m}}$, whereas binary and ternary mixtures with only $15 \%$ CHOL display $\mathrm{L}_{\mathrm{o}} /$ gel coexistence at low $T$, indicated by the presence of convoluted sharp and a broad peaks. These peaks are associated with the melting of CHOL-poor and CHOL-rich environments, ${ }^{13,15}$ and the simulations reveal that on the molecular level the broad peak corresponds to the dissolution of the hexagonal clusters of DPPC chains. 

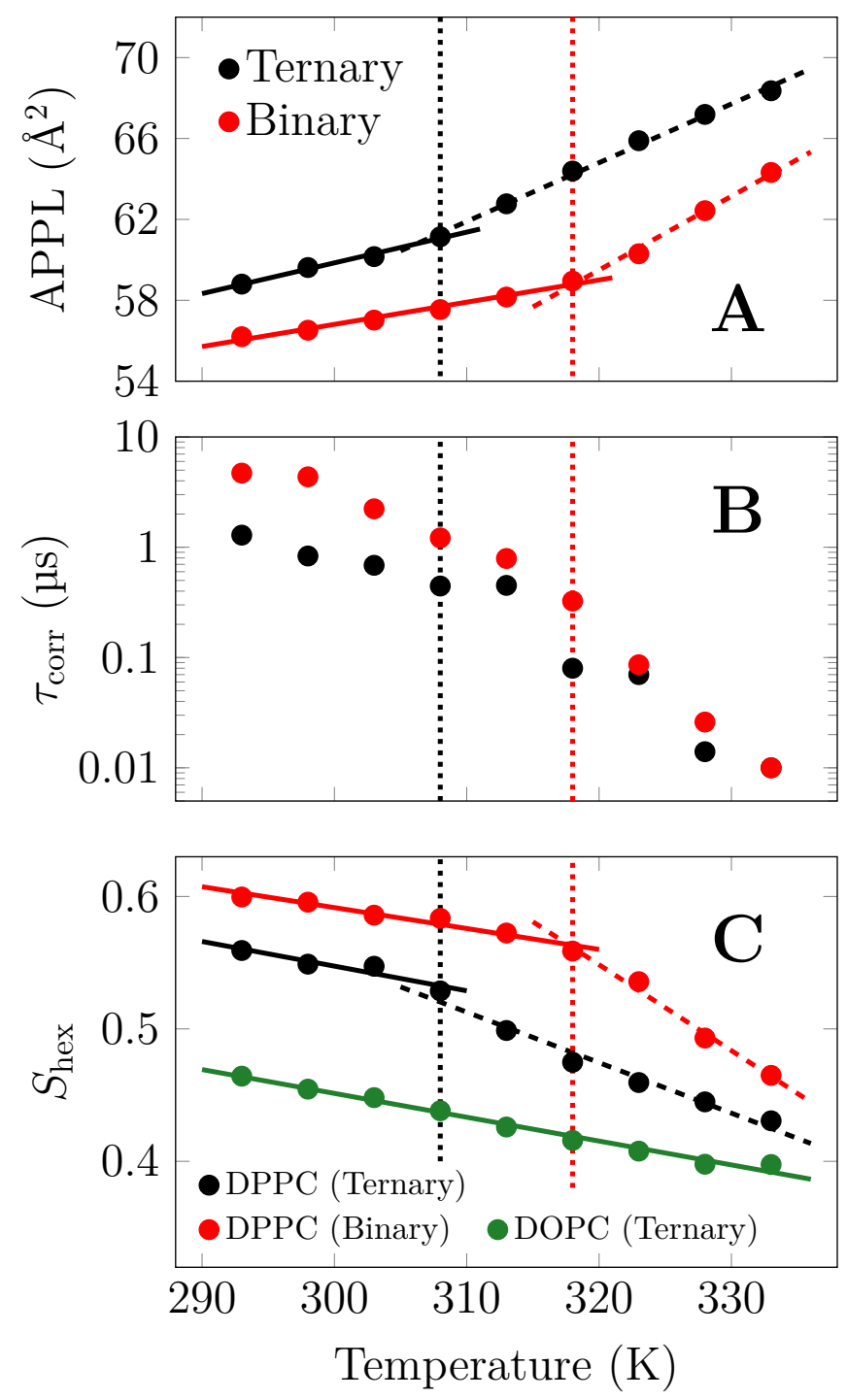

Figure 2: Temperature dependence of membrane properties for the binary and ternary mixtures. A) area per phospholipid (APPL) shows two different slopes, i.e. two different thermal expansion coefficients (solid and dashed lines) with a crossover at either $T_{\mathrm{co}}^{\mathrm{ter}} \approx 308 \mathrm{~K}$ or $T_{\mathrm{co}}^{\mathrm{bin}} \approx 318 \mathrm{~K}$, highlighted by the vertical dotted lines. B) The correlation time of the autocorrelation function of the orientation of the glycerol backbone. The autocorrelation data is shown in Fig. S3. C) The hexatic order parameter of the lipid chains. The parameter values range from 0 to 1 . For DOPC data, no change of slope in the ternary system is observed.

Next, we verified that the membrane really shows $\mathrm{L}_{\mathrm{o}}$-like rapid dynamics on both sides of $T_{\mathrm{co}}$. The characteristic rotational times of DPPC glycerol backbone are shown in Fig. 2B. The rotational times are smaller than those of the gel phase, ${ }^{30}$ and decrease continuously upon heating by two orders of magnitude. They also agree reasonably well with those measured 
for the binary mixture at $285 \mathrm{~K} .{ }^{31}$ The fluid-like nature can also be concluded by comparing lateral diffusion coefficients, shown in Fig. S4, with experimental data in different phases; ${ }^{3}$ The membranes are fluid, yet dynamics speed up significantly above $T_{\mathrm{co}}$. Additionally, we verified that the mean deuterium order parameters show $\mathrm{L}_{\mathrm{o}}$-like high ordering at all studied temperatures (see Fig. S5).

Next, we looked into lipid packing at both sides of $T_{\mathrm{co}}$ in detail. We used the hexatic order parameter $S_{\text {hex }}$ of chosen in-plane atoms in the acyl chain region to characterize the degree of hexagonal packing. ${ }^{21-23}$ As shown in Fig. 2C, DPPC shows relatively high $S_{\text {hex }}$ values in both mixtures until $T_{\mathrm{co}}$, above which they decay fast towards $S_{\text {hex }}$ of DOPC. The values in the binary system are slightly higher as DOPC is not perturbing its packing. DOPC itself is not involved in the hexagonally-packed clusters based on the lack of a crossover in its curve. These findings agree with movies showing the melting of the clusters in the ternary mixture at $T_{\text {co }}^{\text {ter }}$, available at $10.6084 / \mathrm{m}$ 9.figshare.13176167. Lipid exchange rates in these clusters, shown in Fig. S6, also show a crossover at $T_{\text {co. }}$.

We next evaluated whether previously suggested structural changes that take place within $\mathrm{L}_{\mathrm{o}}$ phase ${ }^{13-17}$ are present in our simulations. As shown in Fig. 3A, the mean tilt angle of DPPC chains shows two different slopes with crossovers at $T_{\mathrm{co}}$, in line with the predicted variation in chain tilting within the $\mathrm{L}_{\mathrm{o}}$ region. ${ }^{15}$ Clarke et al. and Reinl et al. suggested that temperature increase within the $\mathrm{L}_{\mathrm{o}}$ phase shifts CHOL towards the headgroup region, leading to the disordering of the lipid chain terminal carbons. ${ }^{16,17}$ This repositioning of CHOL towards the headgroup region indeed takes place (Fig. S7), which further decreases acyl chain ordering in the membrane core. Indeed, as shown in Fig. 3C, the mean deuterium order parameter of the last 9 carbons of the DPPC $s n-2$ chain displays crossovers at $T_{\text {co. }}$. The glycerol region melting, suggested to occur within the $\mathrm{L}_{\mathrm{o}}$ phase by Clarke et al., ${ }^{16}$ also takes place as indicated by the significant decrease in glycerol dynamics in Fig. 2B, yet without a distinguishable crossover.

Next, we categorized the DPPC chains based on their local structure. Our algorithm (see 



Figure 3: Membrane properties which were suggested to differ across temperature in the $\mathrm{L}_{\mathrm{o}}$ regime by earlier experimental studies. A) DPPC chain tilt shows a change in slope at the crossover temperatures of $T_{\text {co }}^{\mathrm{ter}} \approx 308 \mathrm{~K}$ and $T_{\mathrm{co}}^{\mathrm{bin}} \approx 318 \mathrm{~K}$. B) The mean order parameter of all DPPC sn-2 chains, starting from the $8^{\text {th }}$ carbon, i.e. the middle of the chain. The carbonwise order parameter plots are shown in Fig. S5. C) Mean order parameter of DPPC chains as a function of cluster identity (bars) and the fractions of the different cluster identities (lines). "Core" chains are surrounded by hexagonally-packed chains, "free" chains are not part of a hexagonally-packed cluster, and "edge" points roughly correspond to the cluster edges.

Methods in the SI) distinguishes "core" chains encapsulated in the clusters, "edge" chains mainly at cluster edges, and "free" chains that are excluded from the clusters (see Fig. S8 for demonstration). The mean deuterium order parameter $\left(\overline{S_{\mathrm{CD}}}\right)$, shown in Fig. 3C, demonstrates that "core" chains are the most ordered at all temperatures. Notably, they are also the furthest $(0.90 \mathrm{~nm})$ away from CHOL molecules. The "free" chains are the least ordered and closest to a CHOL (0.62 nm), whereas "edge" chains show intermediate behavior. As shown 
in Fig. S9, the "core" chains are also the least tilted, followed by the "edge" chains and finally the "free" chains. The temperature dependence of both $\overline{S_{\mathrm{CD}}}$ and chain tilt consist of two factors, as demonstrated in Figs. 3C and S9: The "core", "edge", and "free" chain populations depend on temperature with the "core" fraction declining rapidly after $T_{\text {co }}$, corresponding to the melting of the clusters. Moreover, while "free" chains show continuous change in ordering and tilt, there is a crossover in the values of the "core" and "edge" chains at $T_{\text {co. }}$ These two factors contribute to the striking change of slope in the temperature dependence of these properties in Fig. 3.

To provide further experimental proof for the described changes in molecular structure, we performed two complementary fluorescence experiments on unilamellar vesicles. We first utilized Patman, which is a fluorescent probe stably located in all membrane phases that probes the lipid carbonyl region. Upon the excitation of Patman, a charge transfer over the naphthalene ring provides a large change in fluorophore dipole moment, and this Stokes shift provides information on the hydration and mobility of the carbonyl region-both sensitive to the membrane phase. Patman has characteristic emission wavelengths in gel $(420 \mathrm{~nm})$ and $\mathrm{L}_{\mathrm{d}}(495 \mathrm{~nm})$ phases, and the respective intensities are used to define the so-called generalized polarization parameter GP $=\left(F_{420}-F_{495}\right) /\left(F_{420}+F_{495}\right)$. GP contains information on fluidity and polarity, with high GP values associated with gel and $\mathrm{L}_{\mathrm{o}}$ phases, and low ones with the $\mathrm{L}_{\mathrm{d}}$ phase. For details, see the Methods and the SI.

GP of the binary and ternary mixtures, DPPC (gel- $\mathrm{L}_{\mathrm{d}}$ transition at $314 \mathrm{~K}$, taken from Ref. 32), and POPC ( $\mathrm{L}_{\mathrm{d}}$ across all measured temperatures), are shown in Fig. 4B with the spectra in Fig. S12. In all systems, the emitted wavelengths red-shift gradually when temperature increases, reflecting an increased mobility of the lipid carbonyls. For the $\mathrm{L}_{\mathrm{d}^{-}}$ phase POPC, the effect saturates at higher temperatures, whereas for DPPC, the transition to the gel phase is evident by saturation of GP at below $314 \mathrm{~K}$. Neither of these effects is present in the binary or ternary mixtures. Instead, the curves indicate two intermediate types of behavior. Indeed, fits of modified Boltzmann sigmoidal functions (see the SI) display 

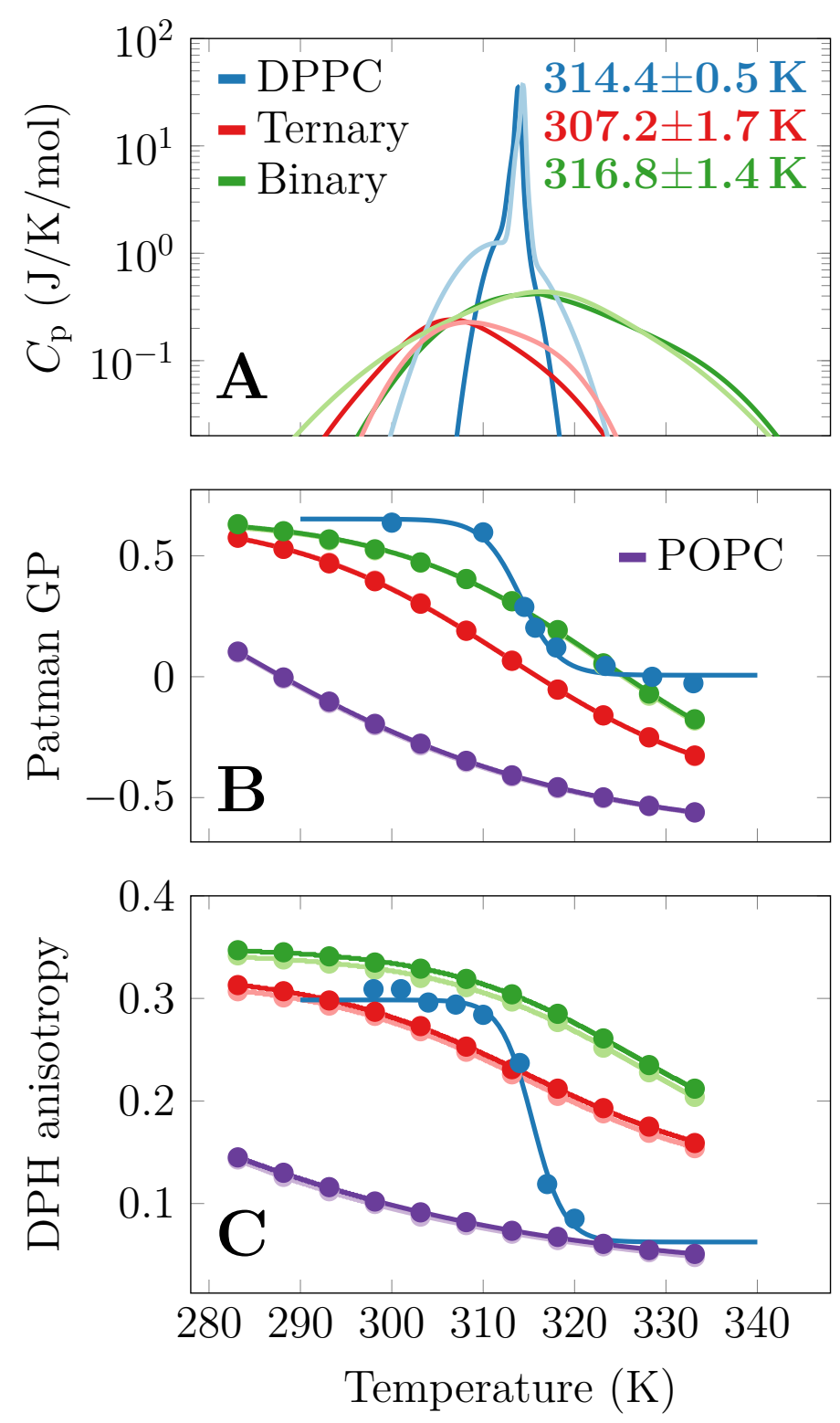

Figure 4: Results from differential scanning calorimetry and fluorescence spectroscopy. A) Specific heat capacity. The profiles were fitted by 2 or 3 Gaussians, and the fitted data are shown here with logarithmic $y$ axis for easier comparison. The original data as well as the fits are shown in Fig. S11, and the parameters obtained from the analysis of the DSC curves are available in Table S2. B) Generalized polarization parameter with Patman. Two independent measurements were performed for each sample yet they overlap. The data for binary and ternary mixtures show a change of curvature (inflection point), yet POPC lacks it, as it is in the $\mathrm{L}_{\mathrm{d}}$ phase at all measured temperatures. The spectra are shown in Fig. S12 and the fitting parameters in Table S3. The absolute values of GP for DPPC, taken from Ref. 32 , should not be directly compared to our data as they use a slightly different definition for GP. C) DPH anisotropy. Two samples were again measured, and they are almost identical. Data for DPPC was taken from Ref. 33. The spectra are shown in Fig. S13 and the fitting parameters in Table $\mathrm{S} 4$. 
that the data for the binary and ternary mixtures display negative (like POPC) and positive (unlike DPPC nor POPC) curvatures with crossovers not far from $T_{\text {co }}$ extracted from our simulations and DSC (see Table S3 for all fitting parameters).

Next, we studied the hydrocarbon chain region with diphenylhexatriene (DPH), which is a rod-like hydrophobic fluorescent probe with simple geometry and directional emission of polarized light. It orients along the lipid hydrocarbon chains and measures their ordering and dynamics - both indicative of the membrane phase. Our previous studies using timeresolved anisotropy measurements demonstrated great sensitivity of DPH to the ordering effect of different amounts and different species of sterols in lipid bilayer. ${ }^{34,35}$

We measured the temperature-dependence of steady-state anisotropy emission spectra of DPH embedded in unilamellar vesicles. The anisotropy values obtained by averaging the spectra (Fig. S13) are shown in Fig. 4C. They show a gradual decrease in anisotropy upon temperature increase, reflecting a decreased order and/or increased dynamics of the lipid aliphatic tails. Noteworthy, the qualitative similarity of the results obtained using Patman generalized polarization and DPH anisotropy reassures that the observed changes can be detected both in the bilayer core, but also closer to the headgroup region, and are not limited to a single fluorescent probe or detection method used. The data can again be fitted by the modified Boltzmann sigmoidal functions, whose curvatures change sign at similar temperatures as those fitted to the GP data (Fig. 4B). Curiously, Patman reports this to take place at a slightly lower temperature, indicating that the hexagonal packing of DPPC chains initiates from the carbonyl region probed by Patman, before it proceeds to the chain region proved by DPH. All the fitting parameters are provided in Table S4.

To conclude, we have provided a molecular-level view into the structures of the $\mathrm{L}_{\mathrm{o}}$ phase across a range of temperatures in binary DPPC/CHOL mixtures and in ternary DPPC/DOPC/CHOL mixtures. Both mixtures are expected to be in the $\mathrm{L}_{\mathrm{o}}$ phase across the studied temperature range from $293 \mathrm{~K}$ to $333 \mathrm{~K}$. Still, we discovered two different regimes separated by a crossover at either $T_{\mathrm{co}}^{\text {ter }} \approx 308 \mathrm{~K}$ or $T_{\mathrm{co}}^{\text {bin }} \approx 318 \mathrm{~K}$, yet no lipid demixing was 
observed.

The hexagonally-packed clusters of DPPC chains that are present at low temperatures melt upon heating at $T_{\mathrm{co}}$, which leads to a change in the temperature dependencies of many structural and dynamic properties. Interestingly, the dependence below and above the crossover was different for the binary and ternary mixtures, yet they converged towards each other at low and high temperatures. The properties of the binary mixture were more different on the two sides of the crossover temperature, highlighting that DOPC is able to buffer many properties in the ternary mixture. Still, all studied properties change continuously with temperature, and no radical changes akin to a $1^{\text {st }}$ order phase transition were observed.

On the experimental side, both the DSC scans and the fluorescence experiments suggest that a structural transition takes place at temperatures close to $T_{\text {co }}$ obtained from simulations. Moreover, both experimental approaches indicate that this transition is more subtle than a proper $1^{\text {st }}$ order phase transition. While the use of probes in fluorescence experiments, the limitations of the simulation models, and the possible differences in the vesicles generated in two laboratories might lead to small discrepancies in the estimated transition temperatures, all approaches systematically suggest that the $T_{\text {co }}$ values differ by $\approx 10 \mathrm{~K}$ between the ternary binary mixtures.

Curiously, the two very different molecular structures - one with gel-like hexagonally packed DPPC clusters among more fluid regions and the other consisting of a fully fluid structure with only slightly preferred interactions among the components - are both fluid: The characteristic timescales of lipid rotational and translational motions differ by two orders of magnitude and thus all fall within the $\mathrm{L}_{\mathrm{o}}$-like regime between the $\mathrm{L}_{\mathrm{d}}$ and gel phases. The $\mathrm{L}_{\mathrm{o}}$-like high values for the deuterium order parameters are also reproduced by our simulations at all the studied temperatures, i.e. by structures where CHOL either is in direct contact with the chains or excluded from the hexagonally-packed clusters of DPPC chains. Therefore, it seems that the two distinct molecular structures where CHOL has two very different roles - either ordering nearby-residing DPPC chains or lubricating the space between the 
gel-like DPPC-clusters - lead to similar average behavior that are indistinguishable by many experiments.

CHOL induces the $\mathrm{L}_{\mathrm{o}} / \mathrm{L}_{\mathrm{d}}$ coexistence in mixtures with a low- $T_{\mathrm{m}}$ lipid and a high- $T_{\mathrm{m}}$ lipid by melting the gel phase (formed mainly by the high- $T_{\mathrm{m}}$ lipid) that would exist in the mixture in the absence of CHOL. However, this "melting" only corresponds to breaking the hexagonally-packed lipids into smaller and mobile clusters. While this leads to $\mathrm{L}_{\mathrm{o}^{-}}$ like behavior, a large fraction of the lipid chains are still in the gel-like highly ordered yet untilted state. Only above $T_{\text {co }}$ do the hexagonally-packed clusters really melt, leading to proper lipid mixing. Curiously, coexisting $\mathrm{L}_{\mathrm{o}}$ and $\mathrm{L}_{\mathrm{d}}$ phases mix in phase-separated DPPC/DOPC/CHOL liposomes at $308 \mathrm{~K}\left(=T_{\mathrm{co}}^{\text {ter }}\right) \cdot{ }^{26}$ Thus, it seems likely that CHOL requires the hexagonally-packed clusters to sustain the $\mathrm{L}_{\mathrm{o}} / \mathrm{L}_{\mathrm{d}}$ coexistence. Indeed, CHOL cannot induce this coexistence from any uniform $\mathrm{L}_{\mathrm{d}}$-phase mixture above $T_{\mathrm{m}}$ of the high- $T_{\mathrm{m}}$ lipid, supporting this idea.

The differences between the two molecular level structures of the $\mathrm{L}_{\mathrm{o}}$ phase in these two temperature regimes might play key roles in biomembranes. Importantly, based on our findings, the use of $\mathrm{L}_{\mathrm{o}} / \mathrm{L}_{\mathrm{d}}$ coexistence observed in synthetic or giant plasma membrane vesicles at relatively low temperatures as a model for lipid rafts existing at body temperature should be questioned, as the structures of the ordered domains in these systems are likely very different.

\section{Acknowledgement}

We thanks CSC-IT Center for Science for computational resources. I.S. thanks the Harvey M. Kruger center for Nanoscience \& Nanotechnology (The Hebrew University) for their fellowship. I.S., B.F. P.J., and M.J. acknowledge the support from the Czech Science Foundation (EXPRO Grant 19-26854X). R.O.P. thanks the Eye and Tissue Bank Foundation, the Mary and Georg C. Ehrnrooth Foundation, and the Evald and Hilda Nissi Foundation 
for financial support. M.J. thanks the academy of Finland (Postdoctoral grant no. 338160) the Emil Aaltonen foundation for funding.

\section{Supporting Information Available}

Simulation inputs and outputs are available at the Zenodo repository at DOI: 10.5281/zenodo.4110687 and DOI: 10.5281/zenodo.4309026. Details on the analyses are provided in the SI and implementations are available from authors upon request.

\section{References}

(1) Ipsen, J. H.; Karlström, G.; Mourtisen, O.; Wennerström, H.; Zuckermann, M. Phase Equilibria in the Phosphatidylcholine-Cholesterol System. BBA-Biomembranes 1987, 905, 162-172.

(2) McMullen, T. P.; Lewis, R. N.; McElhaney, R. N. Cholesterol-Phospholipid Interactions, The Liquid-Ordered Phase and Lipid Rafts in Model and Biological Membranes. Curr. Opin. Colloid Interface Sci. 2004, 8, 459-468.

(3) Rubenstein, J. L.; Smith, B. A.; McConnell, H. M. Lateral Diffusion in Binary Mixtures of Cholesterol and Phosphatidylcholines. Proc. Natl. Acad. Sci. USA 1979, 76, 15-18.

(4) Mills, T. T.; Huang, J.; Feigenson, G. W.; Nagle, J. F. Effects of Cholesterol and Unsaturated DOPC Lipid on Chain Packing of Saturated Gel-phase DPPC Bilayers. Gen. Physiol. Biophys. 2009, 28, 126.

(5) Gallová, J.; Uhríková, D.; Kučerka, N.; Doktorovová, S.; Funari, S. S.; Teixeira, J.; Balgavy, P. The Effects of Cholesterol and $\beta$-sitosterol on the Structure of Saturated Diacylphosphatidylcholine Bilayers. Eur. Biophys. J. 2011, 40, 153-163. 
(6) Vega, M.; Lurio, L.; Lal, J.; Karapetrova, E. A.; Gaillard, E. R. Structure of Supported DPPC/Cholesterol Bilayers Studied via X-ray Reflectivity. Phys. Chem. Chem. Phys. 2020, 22, 19089-19099.

(7) Melchior, D. L.; Scavitto, F. J.; Steim, J. M. Dilatometry of Dipalmitoyllecithin-cholesterol Bilayers. Biochemistry 1980, 19, 4828-4834.

(8) Vist, M. R.; Davis, J. H. Phase Equilibria of Cholesterol/Dipalmitoylphosphatidylcholine Mixtures: Deuterium Nuclear Magnetic Resonance and Differential Scanning Calorimetry. Biochemistry 1990, 29, 451-464.

(9) Zhang, Y.; Lervik, A.; Seddon, J.; Bresme, F. A Coarse-grained Molecular Dynamics Investigation of the Phase Behavior of DPPC/cholesterol Mixtures. Chem. Phys. Lipids 2015, 185, 88-98.

(10) Arnarez, C.; Webb, A.; Rouvière, E.; Lyman, E. Hysteresis and the Cholesterol Dependent Phase Transition in Binary Lipid Mixtures with the Martini Model. J. Phys. Chem. B 2016, 120, 13086-13093.

(11) Waheed, Q.; Tjörnhammar, R.; Edholm, O. Phase Transitions in Coarse-Grained Lipid Bilayers Containing Cholesterol by Molecular Dynamics Simulations. Biophys. J. 2012, 103, 2125-2133.

(12) Wang, Y.; Gkeka, P.; Fuchs, J. E.; Liedl, K. R.; Cournia, Z. DPPC-cholesterol Phase Diagram Using Coarse-grained Molecular Dynamics Simulations. BBA-Biomembranes 2016, 1858, 2846-2857.

(13) McMullen, T. P.; Lewis, R. N.; McElhaney, R. N. Differential Scanning Calorimetric Study of the Effect of Cholesterol on the Thermotropic Phase Behavior of a Homologous Series of Linear Saturated Phosphatidylcholines. Biochemistry 1993, 32, 516-522. 
(14) Huang, T.; Lee, C.; Das Gupta, S.; Blume, A.; Griffin, R. A Carbon-13 and Deuterium Nuclear Magnetic Resonance Study of Phosphatidylcholine/Cholesterol Interactions: Characterization of Liquid-Gel Phases. Biochemistry 1993, 32, 13277-13287.

(15) McMullen, T. P.; McElhaney, R. N. New Aspects of the Interaction of Cholesterol with Dipalmitoylphosphatidylcholine Bilayers as Revealed by High-sensitivity Differential Scanning Calorimetry. BBA-Biomembranes 1995, 1234, 90-98.

(16) Clarke, J. A.; Heron, A. J.; Seddon, J. M.; Law, R. V. The Diversity of the Liquid Ordered $\left(\mathrm{L}_{\mathrm{o}}\right)$ Phase of Phosphatidylcholine/cholesterol Membranes: A Variable Temperature Multinuclear Solid-state NMR and X-ray Diffraction Study. Biophys. J. 2006, 90, 2383-2393.

(17) Reinl, H.; Brumm, T.; Bayerl, T. M. Changes of the Physical Properties of the LiquidOrdered Phase with Temperature in Binary Mixtures of DPPC with Cholesterol: a 2H-NMR, FT-IR, DSC, and Neutron Scattering Study. Biophys. J. 1992, 61, 10251035.

(18) Marsh, D. Cholesterol-induced Fluid Membrane Domains: A Compendium of Lipid-raft Ternary Phase Diagrams. BBA-Biomembranes 2009, 1788, 2114-2123.

(19) Simons, K.; Ikonen, E. Functional Rafts in Cell Membranes. Nature 1997, 387, 569-572.

(20) Baumgart, T.; Hammond, A. T.; Sengupta, P.; Hess, S. T.; Holowka, D. A.; Baird, B. A.; Webb, W. W. Large-Scale Fluid/Fluid Phase Separation of Proteins and Lipids in Giant Plasma Membrane Vesicles. Proc. Natl. Acad. Sci. 2007, 104, 3165-3170.

(21) Sodt, A. J.; Sandar, M. L.; Gawrisch, K.; Pastor, R. W.; Lyman, E. The Molecular Structure of the Liquid-Ordered Phase of Lipid Bilayers. J. Am. Chem. Soc. 2014, 136, $725-732$. 
(22) Gu, R.-X.; Baoukina, S.; Tieleman, D. P. Phase Separation in Atomistic Simulations of Model Membranes. J. Am. Chem. Soc. 2020, 142, 2844-2856.

(23) Javanainen, M.; Martinez-Seara, H.; Vattulainen, I. Nanoscale Membrane Domain Formation Driven by Cholesterol. Sci. Rep. 2017, \%, 1-10.

(24) Wang, C.; Krause, M. R.; Regen, S. L. Push and Pull Forces in Lipid Raft Formation: The Push Can Be as Important as the Pull. J. Am. Chem. Soc. 2015, 137, 664-666.

(25) Löser, L.; Saalwächter, K.; Ferreira, T. M. Liquid-liquid Phase Coexistence in Lipid Membranes Observed by Natural Abundance ${ }^{1} \mathrm{H}-{ }^{13} \mathrm{C}$ Solid-state NMR. Phys. Chem. Chem. Phys. 2018, 20, 9751-9754.

(26) Heftberger, P.; Kollmitzer, B.; Rieder, A. A.; Amenitsch, H.; Pabst, G. In Situ Determination of Structure and Fluctuations of Coexisting Fluid Membrane Domains. Biophys. J. 2015, 108, 854-862.

(27) Veatch, S. L.; Soubias, O.; Keller, S. L.; Gawrisch, K. Critical Fluctuations in DomainForming Lipid Mixtures. Proc. Natl. Acad. Sci. USA 2007, 104, 17650-17655.

(28) Davis, J. H.; Clair, J. J.; Juhasz, J. Phase Equilibria in DOPC/DPPC-d62/Cholesterol Mixtures. Biophys. J. 2009, 96, 521-539.

(29) Krivanek, R.; Okoro, L.; Winter, R. Effect of Cholesterol and Ergosterol on the Compressibility and Volume Fluctuations of Phospholipid-Sterol Bilayers in the Critical Point Region: A Molecular Acoustic and Calorimetric Study. Biophys. J. 2008, 94, $3538-3548$.

(30) Marsh, D. Molecular Motion in Phospholipid Bilayers in the Gel Phase: Long Axis Rotation. Biochemistry 1980, 19, 1632-1637.

(31) Marsh, D.; Watts, A. Molecular Motion in Phospholipid Bilayers in the Gel Phase: 
Spin Label Saturation Transfer ESR Studies. Biochem. Biophys. Res. Commun. 1980, 94, 130-137.

(32) Vaughn, A. R.; Bell, T. A.; Gibbons, E.; Askew, C.; Franchino, H.; Hirsche, K.; Kemsley, L.; Melchor, S.; Moulton, E.; Schwab, M. et al. Relationships Between Membrane Water Molecules and Patman Equilibration Kinetics at Temperatures Far Above the Phosphatidylcholine Melting Point. BBA-Biomembranes 2015, 1848, 942-950.

(33) Neunert, G.; Tomaszewska-Gras, J.; Siejak, P.; Pietralik, Z.; Kozak, M.; Polewski, K. Disruptive Effect of Tocopherol Oxalate on DPPC Liposome Structure: DSC, SAXS, and Fluorescence Anisotropy Studies. Chem. Phys. Lipids 2018, 216, 104-113.

(34) Kulig, W.; Jurkiewicz, P.; Olżyńska, A.; Tynkkynen, J.; Javanainen, M.; Manna, M.; Rog, T.; Hof, M.; Vattulainen, I.; Jungwirth, P. Experimental determination and computational interpretation of biophysical properties of lipid bilayers enriched by cholesteryl hemisuccinate. Biochimica et Biophysica Acta (BBA)-Biomembranes 2015, $1848,422-432$.

(35) Kulig, W.; Olżyńska, A.; Jurkiewicz, P.; Kantola, A. M.; Komulainen, S.; Manna, M.; Pourmousa, M.; Vazdar, M.; Cwiklik, L.; Rog, T. et al. Cholesterol under oxidative stress-How lipid membranes sense oxidation as cholesterol is being replaced by oxysterols. Free radical biology and medicine 2015, 84, 30-41. 